\title{
Pricing and Matching with Frictions
}

\section{Kenneth Burdett}

University of Essex

\section{Shouyong Shi}

Queen's University and Indiana University

\section{Randall Wright}

University of Pennsylvania

\begin{abstract}
Suppose that $n$ buyers each want one unit and $m$ sellers each have one or more units of a good. Sellers post prices, and then buyers choose sellers. In symmetric equilibrium, similar sellers all post one price, and buyers randomize. Hence, more or fewer buyers may arrive than a seller can accommodate. We call this frictions. We solve for prices and the endogenous matching function for finite $n$ and $m$ and consider the limit as $n$ and $m$ grow. The matching function displays decreasing returns but converges to constant returns. We argue that the standard matching function in the literature is misspecified and discuss implications for the Beveridge curve.
\end{abstract}

\section{Introduction}

We analyze a market in which $n$ buyers each want to buy one unit and $m$ sellers each want to sell one or more units of an indivisible good.

We have benefited from the input of many people at many conferences and workshops, including Daron Acemoglu, Melvyn Coles, Jan Eeckhout, Jeremy Greenwood, Ian King, Michael Kremer, Ricardo Lagos, George Mailath, Alan Manning, Mike Peters, Andy Postlewaite, and Rob Shimer. The editor and an anonymous referee also made some excellent comments. The National Science Foundation and the Social Sciences and Humanities Research Council of Canada provided financial support.

[Journal of Political Economy, 2001, vol. 109, no. 5]

(C) 2001 by The University of Chicago. All rights reserved. 0022-3808/2001/10905-0008 $\$ 02.50$

1060 
First sellers set prices, and then each buyer chooses which seller to visit. There is no search problem in the traditional sense because buyers know the price and the capacity of each seller with certainty. ${ }^{1}$ Still, in equilibrium, there is a chance that more buyers will show up at a given location than the seller can accommodate, in which case some customers get rationed; simultaneously, fewer buyers may show up at another location than the seller there can accommodate, in which case the seller gets rationed. This is what we call frictions. We are interested in the relationship between these frictions and pricing decisions and in the number of successful matches between buyers and sellers.

We derive the closed form for the equilibrium prices and matching function with the numbers of buyers and sellers, $n$ and $m$, as arguments and compare the results to predictions of other models. In the case of homogeneous sellers, for example, in a symmetric equilibrium, they all charge the same price $p$, and all buyers choose a seller at random (in some sense endogenizing the meeting process that is simply assumed in the more traditional search literature). In the limit, as $n$ and $m$ get big, $p$ converges to the price generated by a simplified version of the model that is standard in the literature. However, for finite $n$ and $m$, the standard version does not give the correct answer. In terms of our endogenous matching function, for finite $n$ and $m$, it exhibits decreasing returns to scale-that is, frictions get worse as the market gets thicker-but as the market grows, it converges to a function with constant returns.

Sellers here can be thought of as offering combinations of a price and a probability of service. There is much related work in the literature; examples include Butters (1977), Montgomery (1991), Peters (1991, 2000), McAfee (1993), Burdett and Mortensen (1998), Coles and Eeckhout $(2000 a, 2000 b)$, Lagos (2000), and Mortensen and Wright (in press). Indeed, our model with two sellers and two buyers is isomorphic to Montgomery's model, where he has two workers and two firms. However, the analyses differ in our $n \times m$ model and the $n \times m$ version of his model. Intuitively, we take into account explicitly the strategic interaction between sellers, whereas previous analyses following Montgomery assume that each firm sets a price or wage taking as given some measure of aggregate market conditions (see also Lang 1991; Shimer 1996; Moen 1997; Acemoglu and Shimer 1999a, 1999b). Again, the two methods give different answers for finite $n$ and $m$, but we show that they converge to the same limit as the market grows.

We also allow sellers to differ in capacity, exogenously in one version of the model and endogenously in another version. This leads to several

\footnotetext{
${ }^{1}$ In the literature these days, this is called directed rather than undirected search; see the references below.
} 
new insights. For example, the effect on prices of an increase in supply can be different depending on whether this increase occurs along the intensive or the extensive margin (i.e., a change in the number of goods per seller or a change in the number of sellers). Moreover, we show that the matching function depends not only on the number of buyers and the number of goods for sale but also on the distribution of those goods across sellers. For example, it makes a difference if there is one large seller or many small sellers. As with the effect on prices, we show that the effect of an increase in supply on the number of successful matches in equilibrium can be different depending on whether it occurs along the intensive or the extensive margin.

This suggests that the standard matching function used in the literature is misspecified. In the typical labor market application, as in Pissarides (1990) or Mortensen and Pissarides (1994), for example, job creation depends on the number of unemployed workers and the number of vacancies. Our results imply that it should also depend on whether there are many firms each with a few vacancies or a few firms with many vacancies. This allows us to propose a new explanation of the shifts in the Beveridge curve (the locus of observed points in vacancy-unemployment space) documented by Blanchard and Diamond (1989) and Jackman, Layard, and Pissarides (1989). Our explanation is that these shifts may be due to changes in the firm-size distribution. In fact, the relative number of small firms has increased over time (Stanworth and Gray 1991), which in our model would imply the observed shifts in the Beveridge curve.

The rest of the paper is organized as follows. Section II examines the case of two homogeneous buyers and two sellers, each with unit capacity. Section III presents results for the case with $n$ buyers and $m$ sellers, still with unit capacity. Section IV describes the alternative and more standard method for analyzing these kinds of models and shows that it gives the wrong answer for finite $n$ and $m$ but the right answer in the limit. Section $\mathrm{V}$ extends the framework to allow sellers to differ in capacity and discusses the difference between changes in supply along the intensive and extensive margins in terms of the implications for prices and the matching function. Section VI presents some brief concluding remarks.

\section{The $2 \times 2$ Case}

In general there are $n$ buyers and $m$ sellers, but we begin with $n=$ $m=2$. Label the buyers 1 and 2 and the sellers $A$ and $B$. Each buyer wants to buy one unit of an indivisible good and is willing to pay up to his reservation price, which is normalized to one. If he buys at price $p$, he obtains utility $u=1-p$; if he does not buy, he obtains zero. For 
now sellers are homogeneous, and each wants to sell one unit at a price above his reservation price of zero. If he sells at price $p$, he obtains payoff $\pi=p$; if he does not sell, he obtains zero.

The process of exchange proceeds in two stages. First, each seller $j$ posts a price $p_{j}$ taking as given the price of his competitor (more generally, his $m-1$ competitors). At the second stage, each buyer chooses a probability of visiting each seller, taking as given prices and the strategies of other buyers. If two or more buyers show up at the same location, the good is allocated randomly at the posted price. ${ }^{2}$ Let $\theta_{i}$ be the probability that buyer $i$ visits seller $A$ and $1-\theta_{i}$ the probability that he visits $B$. Let $U_{i j}$ be his expected payoff if he visits seller $j$ and $U_{i}=$ $\max \left\{U_{i A}, U_{i B}\right\}$. To compute $U_{i}$, observe that if buyer 1 visits seller $A$, his expected payoff is $1-p_{A}$ times the probability that he gets served. If buyer 2 also visits $A$, which occurs with probability $\theta_{2}$, buyer 1 gets served with probability one-half; if buyer 2 does not visit $A$, buyer 1 gets served for sure. So

$$
U_{1 A}=\left(\frac{\theta_{2}}{2}+1-\theta_{2}\right)\left(1-p_{A}\right)=\frac{1}{2}\left(2-\theta_{2}\right)\left(1-p_{A}\right) .
$$

Similarly, $U_{1 B}=\frac{1}{2}\left(1+\theta_{2}\right)\left(1-p_{B}\right)$. Buyer 2's payoffs are symmetric.

Consider the second-stage game. By comparing $U_{1 A}$ and $U_{1 B}$ taking $\theta_{2}$ and $\left(p_{A}, p_{B}\right)$ as given, we see that the best response of buyer 1 is to go to seller $A$ with probability

$$
\theta_{1}= \begin{cases}0 & \text { if } \theta_{2}>\theta\left(p_{A}, p_{B}\right) \\ 1 & \text { if } \theta_{2}<\theta\left(p_{A}, p_{B}\right) \\ {[0,1]} & \text { if } \theta_{2}=\theta\left(p_{A}, p_{B}\right)\end{cases}
$$

where

$$
\theta\left(p_{A}, p_{B}\right)=\frac{1+p_{B}-2 p_{A}}{2-p_{A}-p_{B}} .
$$

Buyers 2's best response is symmetric. Notice that a buyer does not necessarily go to the seller with a lower price since the probability of rationing needs to be taken into account. In any case, equilibrium in the second-stage game is as follows. First, if $p_{A} \geq\left(1+p_{B}\right) / 2$, the unique equilibrium is $\left(\theta_{1}, \theta_{2}\right)=(0,0)$ (both buyers go to $\left.B\right)$. Second, if $p_{A} \leq$ $2 p_{B}-1$, the unique equilibrium is $\left(\theta_{1}, \theta_{2}\right)=(1,1)$ (both go to $A$ ). Finally, if $2 p_{B}-1<p_{A}<\frac{1}{2}\left(1+p_{B}\right)$, there are exactly three equilibria: two

\footnotetext{
${ }^{2}$ By assumption, a buyer who is rationed cannot sample a second location within the period, but the basic message depends only on there being some cost to doing so. Also, we assume that sellers cannot make price contingent on how many buyers show up. Coles and Eeckhout $(2000 a)$ generalize the model so that sellers are allowed to condition prices on the number of buyers and show that there is always an equilibrium in which prices do not depend on the number of buyers; hence, this restriction is not binding.
} 


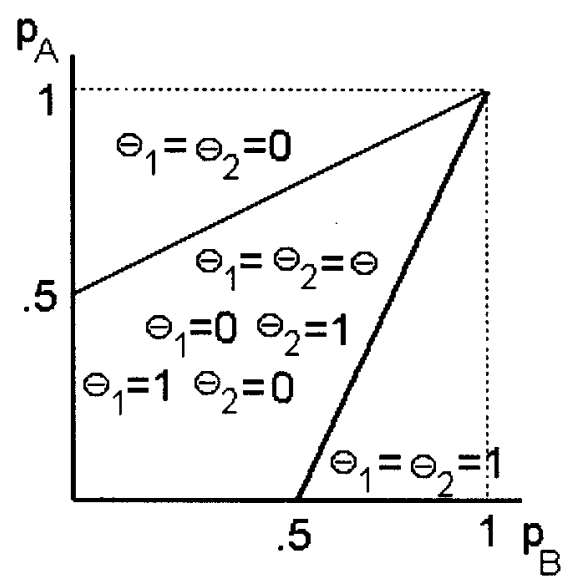

FIG. 1

pure-strategy equilibria, $\left(\theta_{1}, \theta_{2}\right)=(0,1)$ and $\left(\theta_{1}, \theta_{2}\right)=(1,0)$, plus a mixed-strategy equilibrium, $\theta_{1}=\theta_{2}=\theta\left(p_{A}, p_{B}\right)$ (see fig. 1).

Consider now the first-stage game. The expected profit of seller $A$ is as follows. If $p_{A} \leq 2 p_{B}-1$, then $\pi_{A}=p_{A}$ (since he gets both customers for sure); if $p_{A} \geq \frac{1}{2}\left(1+p_{B}\right)$, then $\pi_{A}=0$ (since he gets no customers); and if $2 p_{B}-1<p_{A}<\frac{1}{2}\left(1+p_{B}\right)$, then, since there are multiple equilibria at the second stage, there are two possibilities. If buyers play either of the two pure-strategy equilibria, then $\pi_{A}=p_{A}$; and if buyers play the mixed-strategy equilibrium, then

$$
\pi_{A}=p_{A} \frac{3\left(1-p_{B}\right)\left(1+p_{B}-2 p_{A}\right)}{\left(2-p_{A}-p_{B}\right)^{2}} .
$$

If buyers play the mixed-strategy equilibrium at the second stage, the best response and profit function of seller $A$ to the pricing decision of $B$ are given by

$$
p_{A}^{*}\left(p_{B}\right)=\frac{\left(2-p_{B}\right)\left(1+p_{B}\right)}{7-5 p_{B}}
$$

and

$$
\pi_{A}^{*}\left(p_{B}\right)=\frac{\left(1+p_{B}\right)^{2}}{4\left(2-p_{B}\right)} .
$$

The conditional (on being in the mixed-strategy equilibrium at the 


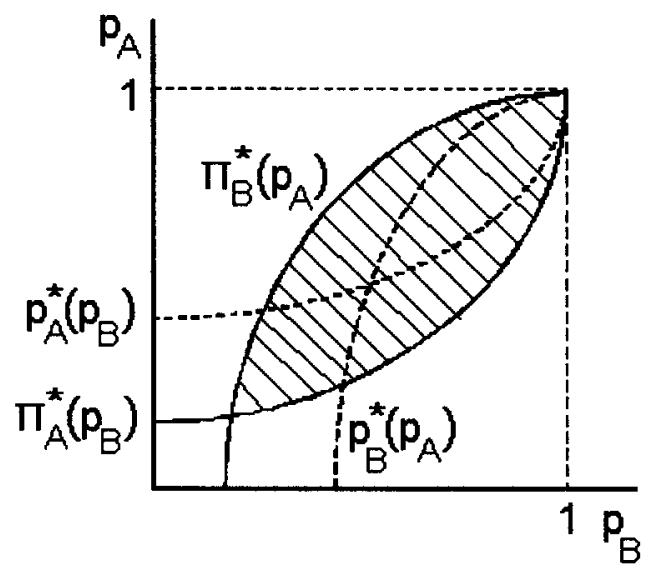

FIG. 2

second stage) best response and profit functions of $B$ are derived symmetrically.

Figure 2 shows the conditional best response and profit functions. It is easily verified that $p_{A}^{*}$ and $\pi_{A}^{*}$ are upward-sloping and convex, and $p_{A}^{*}$ lies above $\pi_{A}^{*}$, as shown. Clearly, $p_{B}^{*}$ and $\pi_{B}^{*}$ are mirror images of $p_{A}^{*}$ and $\pi_{A}^{*}$. Also, $p_{A}^{*}$ and $p_{B}^{*}$ intersect at $\left(\frac{1}{2}, \frac{1}{2}\right)$, whereas $\pi_{A}^{*}$ and $\pi_{B}^{*}$ intersect at $\left(\frac{1}{5}, \frac{1}{5}\right)$. Also, given $p_{B}$, note that if seller $A$ sets $p_{A}=p_{A}^{*}\left(p_{B}\right)$, then his expected profit is no less than $\pi_{A}^{*}\left(p_{B}\right)$, no matter what happens at the second stage: he earns $\pi_{A}=\pi_{A}^{*}\left(p_{B}\right)$ if buyers play the mixed-strategy equilibrium and $\pi_{A}=p_{A}^{*}$ if buyers play a pure-strategy equilibrium. These results are useful for establishing the following proposition.

Proposition 1. A pair $\left(p_{A}, p_{B}\right)$ is an equilibrium in the pricing game iff it is in the shaded region in figure 2 between $p_{A}=\pi_{A}^{*}\left(p_{B}\right)$ and $p_{B}=\pi_{B}^{*}\left(p_{A}\right)$.

Proof. First suppose that $\left(p_{A}, p_{B}\right)$ is such that $p_{A}<\pi_{A}^{*}$. Since expected profit for seller $A$ is no more than $p_{A}$ in any equilibrium and no less than $\pi_{A}^{*}$ in any equilibrium in which he sets $p_{A}^{*}$, he has a profitable deviation (no matter what equilibrium is played at the second stage). Hence, $\left(p_{A}, p_{B}\right)$ cannot be an equilibrium if it is below the $\pi_{A}^{*}$ curve. By symmetry, there is no equilibrium to the left of the $\pi_{B}^{*}$ curve. Therefore, any equilibria must be in the shaded region. We now show that any $\left(p_{A}\right.$, $p_{B}$ ) in this region is an equilibrium, with the following features. At the second stage the buyers play pure strategies along the equilibrium path-that is, on observing $\left(p_{A}, p_{B}\right)$, one buyer goes to seller $A$ and the other goes to $B$ with probability one-and if there is any deviation by 
a seller, at the second stage they play $\theta_{1}=\theta_{2}=1$ if $p_{A} \leq 2 p_{B}-1, \theta_{1}=$ $\theta_{2}=0$ if $p_{A} \geq \frac{1}{2}\left(1+p_{B}\right)$, and $\theta_{1}=\theta_{2}=\theta\left(p_{A}, p_{B}\right)$ if $2 p_{B}-1>p_{A}>\frac{1}{2}(1+$ $\left.p_{B}\right)$, where $\theta\left(p_{A}, p_{B}\right)$ was defined immediately after (1).

Clearly, buyers' strategies constitute equilibrium at the second stage after a deviation (recall fig. 1). If there is no deviation, buyers play pure strategies, which implies $\pi_{A}=p_{A}$ and $\pi_{B}=p_{B}$. Consider a deviation by seller $A$, to $p_{A}^{d}$, say. There are three cases to consider. (1) If $p_{A}^{d} \leq 2 p_{B}-$ 1 , then at the second stage we must have $\theta_{1}=\theta_{2}=1$ and therefore $\pi_{A} \leq 2 p_{B}-1<p_{A}$. This is not a profitable deviation. (2) If $p_{A}^{d} \geq \frac{1}{2}(1+$ $\left.p_{B}\right)$, then $\theta_{1}=\theta_{2}=0$ and therefore $\pi_{A}=0<p_{A}$. This is not a profitable deviation. (3) If $2 p_{B}-1<p_{A}^{d}<\frac{1}{2}\left(1+p_{B}\right)$, then $\theta_{1}=\theta_{2}=\theta\left(p_{A}^{d}\right.$, $\left.p_{B}\right)$. The best deviation of this sort is $p_{A}^{d}=\left[\left(2-p_{B}\right)\left(1+p_{B}\right)\right] /\left(7-5 p_{B}\right)$, which generates profits $\pi_{A}<p_{A}$. Hence, there is no profitable deviation for seller $A$. By symmetry there is no profitable deviation for $B$. This completes the proof. Q.E.D.

The set of equilibria can be partitioned into the case in which $\left(p_{A}, p_{B}\right)=\left(\frac{1}{2}, \frac{1}{2}\right)$ and buyers play mixed strategies at the second stage, and a large set of prices $\left(p_{A}, p_{B}\right)$ in which buyers play pure strategies and each goes to a different seller with probability one. ${ }^{3}$ All of these except $p_{A}=p_{B}=1$ are sustained by an implicit threat from the market: starting at any $\left(p_{A}, p_{B}\right)$ in the relevant region, if a seller changes his price, buyers trigger to the mixed-strategy equilibrium. Since the mixedstrategy equilibrium is bad for sellers, they will not deviate by adjusting their price. The case $p_{A}=p_{B}=1$ is different. In this case, if a seller deviates by lowering his price by any $\epsilon>0$, both customers come to him with probability one; there is no incentive for him to do so, however, since he is already getting one buyer with probability one, which exhausts his capacity.

All these pure-strategy equilibria require a lot of coordination, in the sense that every buyer has to somehow know where every other buyer is going. This may not be so unreasonable when $n=m=2$, but it seems hard to imagine for general $n$ and $m$, which is what we want to consider below. Moreover, in the equilibria supported by threats, buyers have to also coordinate on where to trigger after a deviation, which is even harder to imagine in a large market. The case $p_{A}=p_{B}=1$ does not have this latter difficulty since it is not supported by triggers; however, this case is not particularly robust. Suppose, as a simple example, that we introduce just a little noise: when seller $j$ tries to set $p_{j}$, the price that actually gets posted is uniformly distributed on $\left(p-\epsilon_{j}, p+\epsilon_{j}\right)$. For arbitrarily small $\epsilon_{B}>0$, it is not an equilibrium for seller $A$ to set $p_{A}=1$

\footnotetext{
${ }^{3}$ Note that the set of equilibrium prices is larger than the area between the conditional best-response functions in fig. 2; in fact, it is the area between the profit functions.
} 
because with probability one-half we have $p_{B}<1$, and this implies $\pi_{A}=0$.

The only other equilibrium is the one in which at the second stage each buyer picks a location at random and $p_{A}=p_{B}=\frac{1}{2}$, as shown by the intersection of the conditional best response functions in figure 2 . This equilibrium requires no coordination whatsoever; indeed, since buyers pick sellers at random, this in some sense endogenizes the meeting process that is simply assumed in the standard undirected search literature. Another property of this equilibrium is that it is the unique symmetric equilibrium (symmetric in the sense that $\theta_{1}=\theta_{2}$ and $p_{A}=$ $\left.p_{B}\right)$. Also, this equilibrium is robust to perturbations such as introducing noise. All these considerations, as well as the fact that it generates interesting implications, suggest that in the general models presented below, it is worth concentrating on symmetric equilibria in which buyers randomize.

We say that these equilibria are characterized by frictions in the sense that with positive probability one seller gets more customers whereas another gets fewer than he can service (Lagos [2000], e.g., uses the term similarly). Of course, an equilibrium with frictions fails to maximize the total available surplus: in this example, the number of possible successful matches is two, but the expected number in the mixed-strategy equilibrium is 1.5. Notice, however, that the equilibrium actually does remarkably well, in the following sense. Given that both buyers go to seller $A$ with some arbitrary probability $\theta$, the probability that two will show up at the same location is $\theta^{2}+(1-\theta)^{2} \geq \frac{1}{2}$. In equilibrium, $\theta^{*}=\frac{1}{2}$, which minimizes this probability. Also notice that, though the total surplus is lower, buyers actually do better in this equilibrium than they do in the only other equilibria that are not supported by triggers, that is, those with $p_{A}=p_{B}=1$, where $U_{A}=U_{B}=0$.

\section{The $n \times m$ Case}

Suppose now that there are $n$ buyers and $m$ sellers. As discussed above, we focus on symmetric equilibria in which all sellers charge the same price and all buyers use the same mixed strategy. Given that all sellers post the same $p$, the mixed strategy each buyer uses must be to visit all sellers with equal probability, $\theta=1 / m$, since otherwise a given buyer would deviate by going to a seller that has the lowest expected number of customers. We have the following results.

Proposition 2. The unique symmetric equilibrium has every buyer visit each seller with probability $\theta^{*}=1 / m$, and all sellers set 


$$
p^{*}=p(m, n)=\frac{m-m\{1+[n /(m-1)]\}[1-(1 / m)]^{n}}{m-\{m+[n /(m-1)]\}[1-(1 / m)]^{n}} .
$$

Proof. To begin, let $\Phi$ be the probability that at least one buyer visits a particular seller when all buyers visit him with the same (but arbitrary) probability $\theta$. Since $(1-\theta)^{n}$ is the probability that all $n$ buyers go elsewhere, $\Phi=1-(1-\theta)^{n}$. Next, let $\Omega$ be the probability that a given buyer gets served when he visits this seller. Since the probability of getting served conditional on visiting this seller times the probability that this buyer visits him equals the probability that this seller serves the particular buyer, we have $\Omega \theta=\Phi / n$. Hence

$$
\Omega=\frac{\Phi}{n \theta}=\frac{1-(1-\theta)^{n}}{n \theta} .
$$

Now suppose that every seller is posting $p$, and one contemplates deviating to $p^{d}$. Let the probability that any given buyer visits the deviant be $\theta^{d}$. Then the probability that he visits each of the nondeviants is $\left(1-\theta^{d}\right) /(m-1)$. By $(6)$, a buyer who visits the deviant gets served with probability

$$
\Omega^{d}=\frac{1-\left(1-\theta^{d}\right)^{n}}{n \theta^{d}}
$$

and a buyer who visits a nondeviant gets served with probability

$$
\Omega=\frac{1-\left\{1-\left[\left(1-\theta^{d}\right) /(m-1)\right]\right\}^{n}}{n\left[\left(1-\theta^{d}\right) /(m-1)\right]} .
$$

In a symmetric equilibrium in the second-stage game, buyers are indifferent between visiting the deviant and any other seller: $\Omega(1-p)=$ $\Omega^{d}\left(1-p^{d}\right)$. When we insert $\Omega$ and $\Omega^{d}$ and rearrange, this condition becomes

$$
\frac{1-p}{1-p^{d}}=\frac{\left(1-\theta^{d}\right)\left[1-\left(1-\theta^{d}\right)^{n}\right]}{(m-1) \theta^{d}\left(1-\left\{1-\left[\left(1-\theta^{d}\right) /(m-1)\right]\right\}^{n}\right)} \equiv \Psi\left(\theta^{d}\right) .
$$

Notice that $\Psi\left(\theta^{d}\right)$ is a strictly decreasing function with

$$
\begin{aligned}
& \lim _{\theta^{d \rightarrow 0}} \Psi\left(\theta^{d}\right)=\frac{n /(m-1)}{1-\{1-[1 /(m-1)]\}^{n}} \geq 1, \\
& \lim _{\theta^{d} \rightarrow 1} \Psi\left(\theta^{d}\right)=\frac{1}{n} .
\end{aligned}
$$

Hence, whenever $\Psi(1)<(1-p) /\left(1-p^{d}\right)<\Psi(0)$, there is a unique $\theta^{d}=\theta^{d}\left(p^{d}, p\right) \in(0,1)$ that makes buyers indifferent between sellers posting $p$ and $p^{d}$. If $(1-p) /\left(1-p^{d}\right)$ is too small or too big, no $\theta^{d} \in$ 
$(0,1)$ makes buyers indifferent, and either all or no buyers visit the deviant. Hence,

$$
\theta^{d}= \begin{cases}0 & \text { if } \frac{1-p}{1-p^{d}}>\Psi(0) \\ 1 & \text { if } \frac{1-p}{1-p^{d}}<\Psi(1) \\ \theta^{d}\left(p^{d}, p\right) & \text { otherwise. }\end{cases}
$$

Expected profit of the deviant is $\pi\left(p^{d}, p\right)=p^{d}\left[1-\left(1-\theta^{d}\right)\right]^{n}$, where $\theta^{d}$ is given by (11). Clearly, the best deviation satisfies $p^{d}>0$ and hence satisfies the first-order condition

$$
\frac{\partial \pi}{\partial p^{d}}=1-\left(1-\theta^{d}\right)^{n}+p^{d} n\left(1-\theta^{d}\right)^{n-1} \frac{\partial \theta^{d}}{\partial p^{d}}=0 .
$$

Assuming $\theta^{d} \in(0,1)$, we can differentiate (11) and then insert the symmetric equilibrium conditions $p^{d}=p$ and $\theta^{d}=1 / m$ to derive

$$
\frac{\partial \theta^{d}}{\partial p^{d}}=\frac{-(m-1)^{2}\left\{1-[(m-1) / m]^{n}\right\}}{m^{2}\left\{(m+n-1)[(m-1) / m]^{n}-m+1\right\}(1-p)} .
$$

Inserting this into (12) and solving, we arrive at (5). As remarked above, given that all sellers post $p$, buyers must visit each with the same probability $\theta^{*}=1 / m$. This completes the proof. Q.E.D.

Figure 3 shows the level sets, or isoprice curves, and a three-dimensional plot of $p^{*}=p(m, n)$. Naturally, price is increasing in $n$ and decreasing in $m$, and $p^{*} \rightarrow 1$ as $n \rightarrow \infty$ whereas $p^{*} \rightarrow 0$ as $m \rightarrow \infty$. Notice that $p^{*}$ does not jump discontinuously from the monopsony price to the monopoly price as the buyer-seller ratio $b=n / m$ crosses 1 , as would be predicted by the simplest frictionless model, so there is a sense in which frictions smooth things out. ${ }^{4}$ One can also ask how the price varies with $m$ when the buyer-seller ratio $b$ is kept fixed. One can verify that $p^{*}$ falls with $m$ in a sellers' market (where $b$ is relatively large) and rises with $m$ in a buyers' market (where $b$ is small); see Cao and Shi (2000) for more discussion. However, in either case, $p$ converges to a very simple limit as the market grows.

Proposition 3. Let $b=n / m$ be fixed. Then the limit of $p(m, n)$ when $m, n \rightarrow \infty$ is

\footnotetext{
${ }^{4}$ By a frictionless model we mean the simplest Walrasian model in which $p$ is zero or one as $n$ is above or below $m$. Of course, some Walrasian models with indivisible goods (and no other frictions) predict that prices vary smoothly with $n$ and $m$ once randomization is introduced via lotteries or sunspots (see, e.g., Rogerson 1988; Shell and Wright 1993).
} 
a

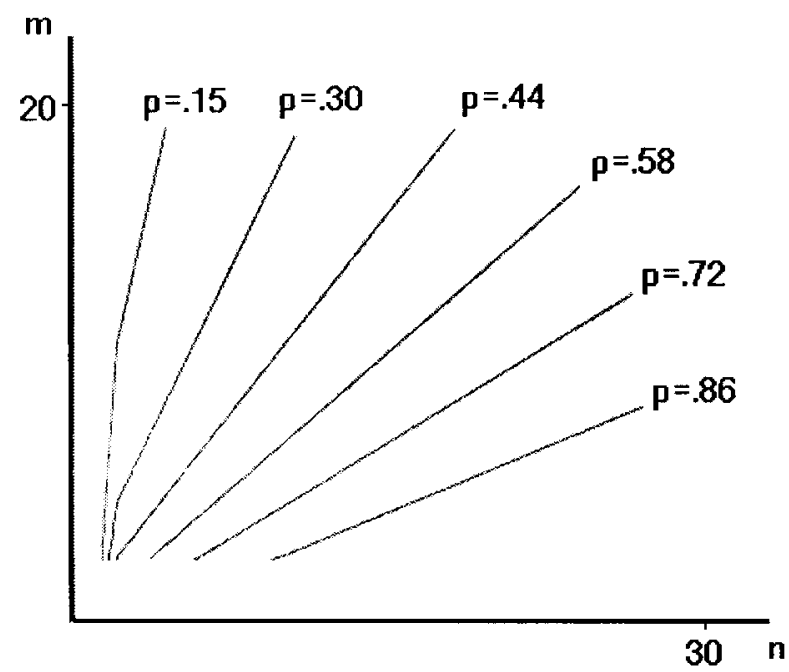

b

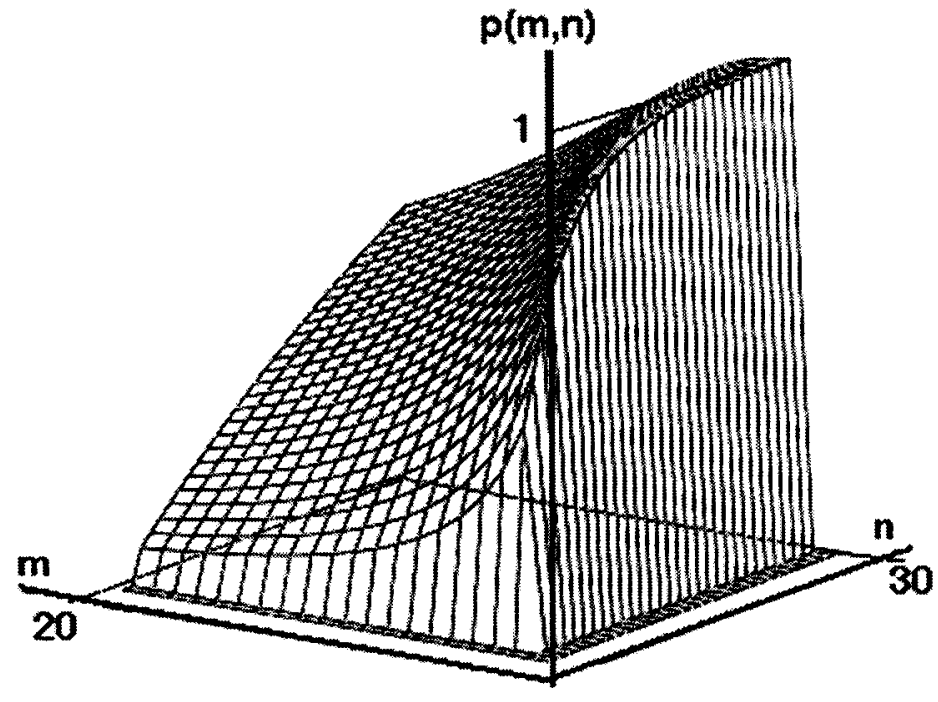

FIG. 3 


$$
\bar{p}=1-\frac{b}{e^{b}-1} .
$$

Proof. Eliminating $n=b m$ from (5), we have

$$
p^{*}=\frac{m-1-[m(b+1)-1][(m-1) / m]^{m b}}{m-1-(m+b-1)[(m-1) / m]^{m b}} .
$$

Taking the limit (which, if one has any trouble, can easily be done using a symbolic package such as Maple) yields the result. Q.E.D.

Given $p(m, n)$, equilibrium values of other variables can now be computed. For example, expected profit and utility are

$$
\pi^{*}=\pi(m, n)=\left[1-\left(1-\frac{1}{m}\right)^{n}\right] p(m, n)
$$

and

$$
U^{*}=U(m, n)=\frac{m}{n}\left[1-\left(1-\frac{1}{m}\right)^{n}\right][1-p(m, n)] .
$$

As $m$ gets large with $b$ held fixed, these converge to $\bar{\pi}=1-(1+$ b) $e^{-b}$ and $\bar{U}=e^{-b}$. We are especially interested in the expected number of sales, or successful buyer-seller matches,

$$
M^{*}=M(m, n)=m\left[1-\left(1-\frac{1}{m}\right)^{n}\right] .
$$

This is the equilibrium matching function, analogous to the specification in search models of the labor market, where the number of worker-firm meetings depends on vacancies and unemployment, as in Pissarides (1990) or Mortensen and Pissarides (1994), for example. Figure 4 shows the level sets, or isomatching curves, and a three-dimensional plot of $M^{*}=M(m, n)$.

The probability of a successful match for an individual, which can be thought of as his arrival rate, is $A_{b}=M(m, n) / n$ for a buyer and $A_{s}=$ $M(m, n) / m$ for a seller. If we fix $n / m=b$, then it is easy to see that the arrival rates are decreasing in $m$. This means that $M(m, n)$ exhibits $d e$ creasing returns to scale or, in other words, that there is more friction in bigger markets. However, $A_{b}$ and $A_{s}$ converge to $\left(1-e^{-b}\right) / b$ and $1-$ $e^{-b}$, which means that we have approximately constant returns, when $m$ is large. Also notice that $M(n, m)$ is not homothetic: as seen in figure 4 , along the ray $b=n / m$, the isomatching curves get steeper (flatter) as $m$ increases when $b$ is large (small). However, the slopes of the isomatching curves converge to $1 /\left(1+b-e^{b}\right)$, which depends only on $b$, and so $M(n, m)$ is approximately homothetic when $m$ is large.

The functional form in (18) suggests an alternative to the standard 

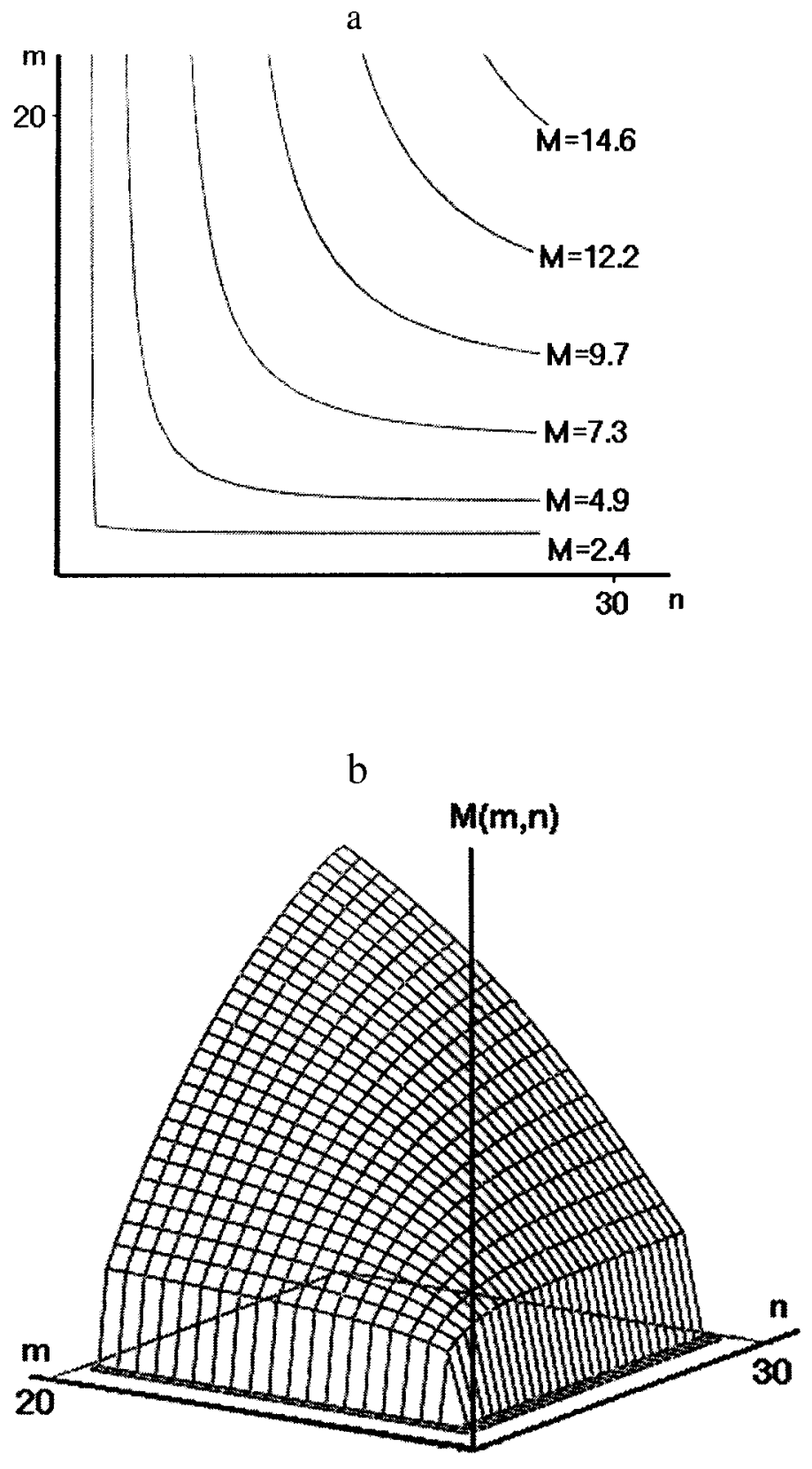

FIG. 4 


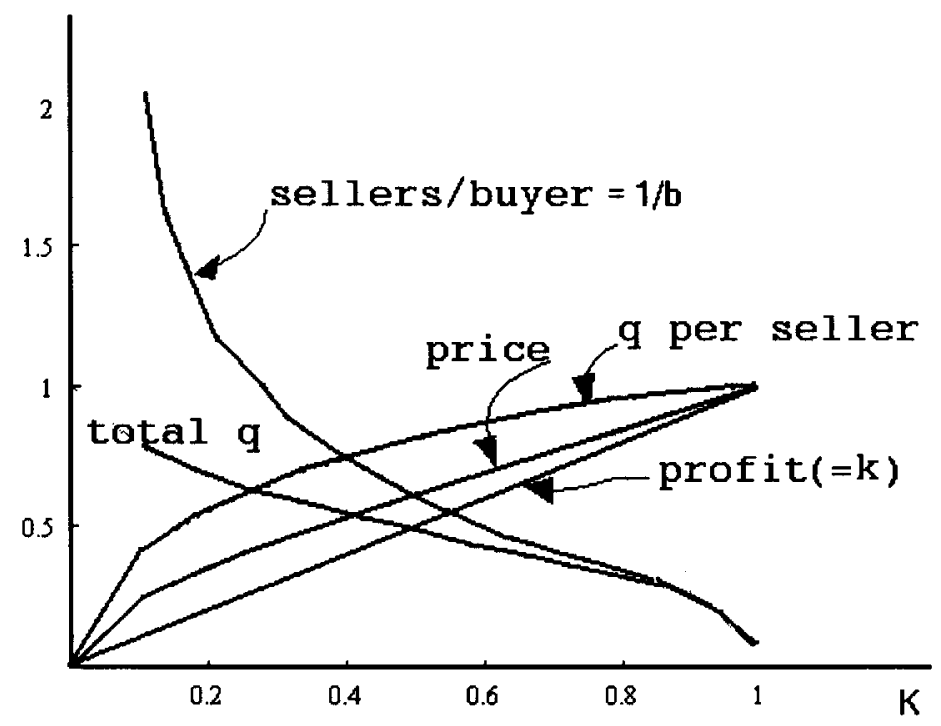

FIG. 5

Cobb-Douglas specification assumed in most applied work, although when we return to matching functions below, we shall argue that one may want to be cautious of any specification of the form $M(n, m)$. In any case, we close the section by mentioning that, while we are on the subject of matching models, one can easily add a Pissarides-style entry condition to the framework. That is, we can determine the number of sellers endogenously by setting $\pi(m, n)=K$, where $K$ is the cost of entry and $\pi(m, n)$ is given by (16). Given $K$ and the number of buyers $n$, entry determines $m$ and thus all endogenous variables, including the buyerseller ratio, price, and expected quantity (see fig. 5).

\section{An Alternative Solution Method}

Here we present the analogue of the approach in Montgomery (1991) and some of the other papers mentioned in the Introduction. The key to this method is to assume that sellers take as given that they must offer buyers a certain level of expected utility $U$, which later we determine endogenously. Thus, suppose that an individual seller chooses $p$ and buyers respond by coming to him with probability $\theta$; then $p$ and $\theta$ must generate an expected utility of at least $U$ if he is to get any customers. Recall from Section III that the probability that a seller gets at 
least one buyer is $1-(1-\theta)^{n}$ and the probability that a buyer who visits him gets served is $\Omega=\left[1-(1-\theta)^{n}\right] / n \theta$; hence, the seller's problem is

$$
\begin{aligned}
& \max \pi=p\left[1-(1-\theta)^{n}\right] \\
& \text { subject to }(1-p) \Omega \geq U .
\end{aligned}
$$

Solving the constraint at equality for $p$, substituting into the objective function, and maximizing with respect to $\theta$, we arrive at

$$
(1-\theta)^{n-1}=U .
$$

Since sellers all take $U$ as given, they all choose the same $\theta$ and $p$, and therefore any possible equilibrium is symmetric and entails $\theta=1 / \mathrm{m}$. Inserting this into (20) determines $U$, and then the constraint in (19) can be solved for

$$
p^{a}=1-\frac{n[1-(1 / m)]^{n-1}}{m\left\{1-[1-(1 / m)]^{n}\right\}} .
$$

This is not the same as (15). If $n=m=2$, for example, (21) yields $p^{a}=\frac{1}{3}$; whereas we know from (5), and also from Section II, that the correct answer is $p^{*}=\frac{1}{2}$.

The problem with the method leading to (21) is that it ignores elements of strategic interaction among sellers. At least for small values of $n$ and $m$, it does not really make sense to take $U$ parametrically because a change in a seller's price implies a change in the probability that buyers visit him, a change in the probability that they visit other sellers, and a change in market utility. Our method takes this into account: in (9) the left-hand side is the expected utility a buyer gets from visiting a deviant seller, the right-hand side is the expected utility a buyer gets from visiting a nondeviant seller, and both sides depend on $\theta^{d}$. This is not the case with the constraint in (19), where the right-hand side is a fixed number $U$ independent of $\theta^{d}$.

Intuitively, our model captures competition for customers among sellers, whereas the method leading to (21) captures only competition between a seller and the market. One might expect this distinction to vanish as the market gets big. This is indeed the case: setting $n=m b$ in (21) and letting $m \rightarrow \infty$, we see that $p^{a} \rightarrow 1-\left[b /\left(e^{b}-1\right)\right]$, which is the same as the value for $\bar{p}$ in (14). In other words, the alternative solution method outlined in this section gives approximately the correct answer in large markets.

\section{Heterogeneous Sellers}

We are interested in what happens where sellers differ. Heterogeneity in terms of a different quality of goods is easy to handle and potentially 
interesting given that consumers would then have to trade off quality as well as price and the probability of service. ${ }^{5}$ However, we are more interested here in sellers that differ in capacity. We shall first illustrate what happens with an example and then proceed to the general case. So, to begin, consider the $2 \times 2$ model in which seller $A$ now has the option of producing a second unit at $\operatorname{cost} c_{2}$, whereas $B$ still has one unit. Assume that $A$ sets the same price for both units. Intuitively, in this case we expect $p_{A}>p_{B}$ since seller $A$ never rations. However, we still expect buyers to go to $B$ with positive probability since he will be cheaper, even though he may ration.

Generalizing Section II, we can easily show that the symmetric equilibrium in the second stage is for both buyers to go to seller $A$ with probability $\theta=\left(1-2 p_{A}+p_{B}\right) /\left(1-p_{B}\right)$. Note that $p_{A}=p_{B}$ implies $\theta=$ 1; $B$ will obviously have to cut his price to compete. The conditional reaction functions are

$$
p_{A}=\frac{p_{B}+1}{4}, \quad p_{B}=\frac{p_{A}}{2-p_{B}} .
$$

The solution is $\left(p_{A}, p_{B}\right)=(.293, .172)$, which implies $\theta=.707$. Expected profit for $A$ is $\pi_{A}=.414-c_{2}$, and since $\pi_{A}=.375$ when $A$ had only one unit of capacity, he is willing to produce the second unit iff $c_{2} \leq .039$. This may seem low, given that $A$ can in principle corner the market with a second unit and given that the good is in principle worth $u=1$ to a buyer; notice, however, that the response of $B$ to capacity expansion by $A$ is a rather drastic price cut.

Now suppose that both sellers choose capacity $k \in\{0,1,2\}$, where the costs of the first and second units are $c_{1}$ and $c_{2}$. Given capacity, they post prices and buyers decide whom to visit, as before. If a symmetric equilibrium is played once $\left(k_{A}, k_{B}\right)$ is determined, payoffs in the capacity game are described by the matrix

\footnotetext{
${ }^{5}$ Consider the $2 \times 2$ case in which the utility from the good of seller $A$ is $u_{A}=\beta$ and the utility from $B$ is $u_{B}=1$. When we generalize the analysis in Sec. II, the symmetric equilibrium in the second stage is for both buyers to go to $A$ with probability $\theta=$ $\left(2 \beta-1+p_{B}-p_{A}\right) /\left(1+\beta-p_{A}-p_{B}\right)$; the conditional reaction functions are

$$
\begin{aligned}
& p_{A}=\frac{\left(2 \beta-1+p_{B}\right)\left(1+\beta-p_{B}\right)}{5+2 \beta-5 p_{B}}, \\
& p_{B}=\frac{\left(2-\beta+p_{A}\right)\left(1+\beta-p_{A}\right)}{2+5 \beta-5 p_{A}} .
\end{aligned}
$$
}

For instance, when $u_{A}$ falls from 1 to $0.67, p_{A}$ falls to $0.29, p_{B}$ increases to 0.57 , and customers respond by visiting the low-quality seller $A$ with probability .42 . 


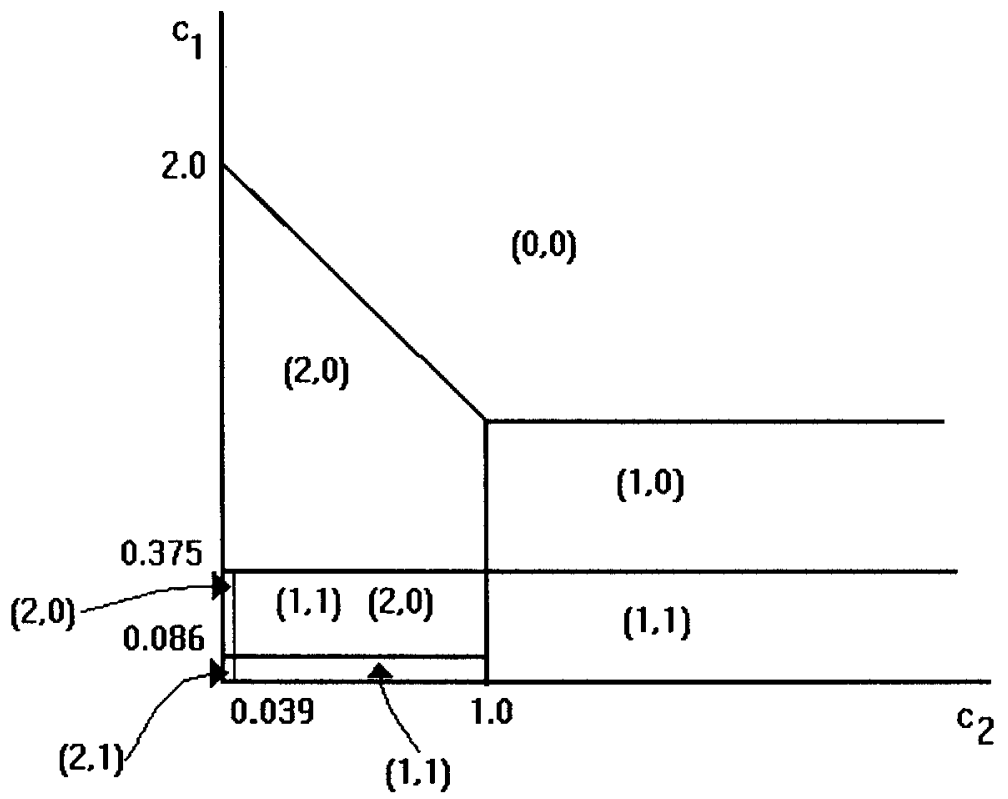

FIG. 6

$$
\left[\begin{array}{ccc}
0,0 & 1-c_{1}, 0 & 2-c_{1}-c_{2}, 0 \\
0,1-c_{1} & .375-c_{1}, .375-c_{1} & .414-c_{1}-c_{2}, .086-c_{1} \\
0,2-c_{1}-c_{2} & .086-c_{1}, .414-c_{1}-c_{2} & -c_{1}-c_{2},-c_{1}-c_{2}
\end{array}\right]
$$

where each position $(i, j)$ lists the payoffs to sellers $A$ and $B$ when $k_{A}=i$ and $k_{B}=j$ for $i, j \in\{0,1,2\}$. Figure 6 shows the equilibria in $\left(c_{1}, c_{2}\right)$ space. ${ }^{6}$ In terms of economic results, we have the following: If $c_{2}$ is much bigger than $c_{1}$, the unique outcome is $\left(k_{A}, k_{B}\right)=(1,1)$; if $c_{2}$ is much smaller than $c_{1}$, then we have $\left(k_{A}, k_{B}\right)=(2,0)$ or $(0,2)$. In the intermediate region these equilibria coexist. We have $\left(k_{A}, k_{B}\right)=$ $(1,0)$ or $(0,1)$ iff $c_{2} \geq 1$ and $c_{1}$ is big. It is never an equilibrium to have $\left(k_{A}, k_{B}\right)=(2,2)$ since this implies $\pi_{A}=\pi_{B}=0$, but we can get $\left(k_{A}, k_{B}\right)=(2,1)$ or $(1,2)$ when $c_{1}$ and $c_{2}$ are small.

It might be interesting to endogenize capacity along these lines in the general case of $n$ buyers and $m$ sellers. However, to focus more clearly on some implications for matching, here we assume exogenously that $m_{L} \in(0, m)$ sellers each have one unit for sale and $m_{H}=m-$

\footnotetext{
${ }^{6}$ To save space, when there are two equilibria that are merely relabelings, such as $\left(k_{A}, k_{B}\right)=(2,0)$ and $\left(k_{A}, k_{B}\right)=(0,2)$, only one is shown in the figure.
} 
$m_{L}$ each have two units. We focus on symmetric equilibria, where all high-capacity sellers charge $p_{H}$ and all low-capacity sellers charge $p_{L}$, and all buyers visit each high-capacity seller with probability $\theta_{H}$ and visit each low-capacity seller with probability $\theta_{L}$. We shall solve the model using the method in Section IV, where sellers take as given the utility $U$ that they must provide buyers. For finite market size we know that this does not give the right answer, but we shall see that it converges to the correct answer as the market gets large, just as in the case of homogeneous sellers.

To begin the analysis, note that a low-capacity seller solves

$$
\begin{aligned}
& \max \pi_{L}=p_{L}\left[1-\left(1-\theta_{L}\right)^{n}\right] \\
& \text { subject to }\left(1-p_{L}\right) \Omega_{L} \geq U,
\end{aligned}
$$

where $\Omega_{L}=\left[1-\left(1-\theta_{L}\right)^{n}\right] / n \theta_{L}$. As this is the same as (19) in the previous section, the solution satisfies

$$
\left(1-\theta_{L}\right)^{n-1}=U \text {. }
$$

For a high-capacity seller, one can show that the relevant problem is

$$
\begin{aligned}
& \max \pi_{H}=p_{H}\left\{2\left[1-\left(1-\theta_{H}\right)^{n}\right]-n \theta_{H}\left(1-\theta_{H}\right)^{n-1}\right\} \\
& \text { subject to }\left(1-p_{L}\right) \Omega_{H} \geq U,
\end{aligned}
$$

where

$$
\Omega_{H}=\frac{2}{n \theta_{H}}\left[1-\left(1-\theta_{H}\right)^{n}\right]-\left(1-\theta_{H}\right)^{n-1}
$$

after a little analysis. ${ }^{7}$ Substituting from the constraint and maximizing with respect to $\theta_{H}$, we have (compare with [24])

${ }^{7}$ A buyer who visits a high-capacity seller gets the good for sure if the seller is visited by either no other buyers or exactly one other buyer, the probability of which is $(1-$ $\left.\theta_{H}\right)^{n-1}+(n-1) \theta_{H}\left(1-\theta_{H}\right)^{n-2}$. If the seller is visited by $k \geq 2$ other buyers, he randomly chooses two to serve, so each buyer gets served with probability $1-\left(C_{k}^{2} / C_{k+1}^{2}\right)=2 /(k+$ $1)$. Thus a buyer who visits a high-capacity seller gets the good with probability

$$
\begin{aligned}
\Omega_{H}= & \left(1-\theta_{H}\right)^{n-1}+(n-1) \theta_{H}\left(1-\theta_{H}\right)^{n-2} \\
& +\sum_{k=2}^{n-1} \frac{2}{k+1} C_{n-1}^{k} \theta_{H}^{k}\left(1-\theta_{H}\right)^{n-1-k} \\
= & \frac{2}{n \theta_{H}}\left[1-\left(1-\theta_{H}\right)^{n}\right]-\left(1-\theta_{H}\right)^{n-1} .
\end{aligned}
$$

Hence, profit for a high-capacity seller is

$$
\pi_{H}=p_{H}\left[n \theta_{H}\left(1-\theta_{H}\right)^{n-1}+2 \sum_{k=2}^{n} C_{n}^{k} \theta_{H}^{k}\left(1-\theta_{H}\right)^{n-k}\right],
$$

where the first term in the brackets is the probability that the seller is visited by only one buyer, and the second is the probability that he is visited by at least two buyers. This simplifies to the expression in the text. 
1078

JOURNAL OF POLITICAL ECONOMY

$$
\left(1-\theta_{H}\right)^{n-1}+\theta_{H}(n-1)\left(1-\theta_{H}\right)^{n-2}=U .
$$

When all sellers were homogeneous, we could insert the result $\theta=$ $1 / m$ into (20) and solve for $U$. Here we have to solve (24), (26), and the adding-up condition $m_{L} \theta_{L}+m_{H} \theta_{H}=1$ simultaneously for $U, \theta_{H}$, and $\theta_{L}$. The result is the following proposition.

Proposition 4. Consider the model with $n$ buyers and $m$ sellers in which $m_{L} \in(0, m)$ sellers have one unit for sale and $m_{H}=m-m_{L}$ have two units, and sellers take $U$ parametrically. Let $b=n / m$ and $h=$ $m_{H} / m_{L}$ be fixed. Then as $n$ and $m$ grow, we have in the limit

$$
p_{L}=1-\frac{b(1-x)}{(1-h)\{\exp [(b-b x) /(1-h)]-1\}}
$$

and

$$
p_{H}=1-\frac{[1+(b x / h)](b x / h)}{2 \exp (b x / h)-2-(b x / h)},
$$

where $x=m_{H} \theta_{H}$. This is the same as the answer one gets by first solving the model for finite $n$ and $m$ taking strategic interaction into account and then taking the limit.

Proof. Combining (24), (26), and the adding-up condition yields

$$
\left(1-\theta_{H}\right)^{n-1}+\theta_{H}(n-1)\left(1-\theta_{H}\right)^{n-2}=\left(1-\frac{1-m_{H} \theta_{H}}{m-m_{H}}\right)^{n-1} .
$$

Rewriting this in terms of $x$, we have

$$
\begin{gathered}
\left(1-\frac{x}{h m}\right)^{b m-1}+\frac{x}{h m}(b m-1)\left(1-\frac{x}{h m}\right)^{b m-2} \\
=\left[1-\frac{1-x}{m(1-h)}\right]^{b m-1} .
\end{gathered}
$$

Letting $m \rightarrow \infty$ and rearranging, we get

$$
1+\frac{b x}{h}=\exp \left[\frac{b(x-h)}{h(1-h)}\right] .
$$

There is a unique $x \in(0,1)$ satisfying $(31)$. Given $x$, we then solve for $U, p_{H}$, and $p_{L}$. From (24) we have

$$
U=\left[1-\frac{1-x}{m(1-h)}\right]^{b m-1} .
$$

Notice that $U \rightarrow \exp [(b x-b) /(1-h)]$ as $m \rightarrow \infty$. From the constraints in (23) and (25) we have 
a

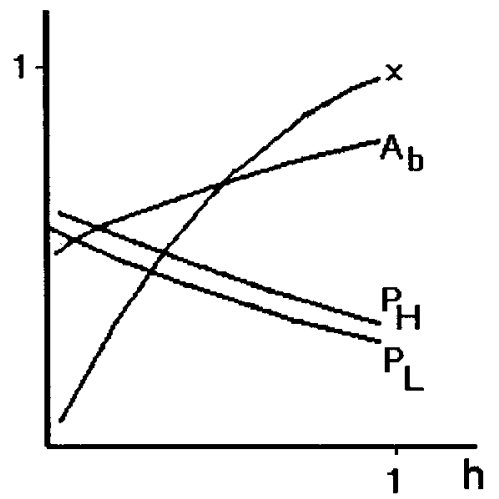

b

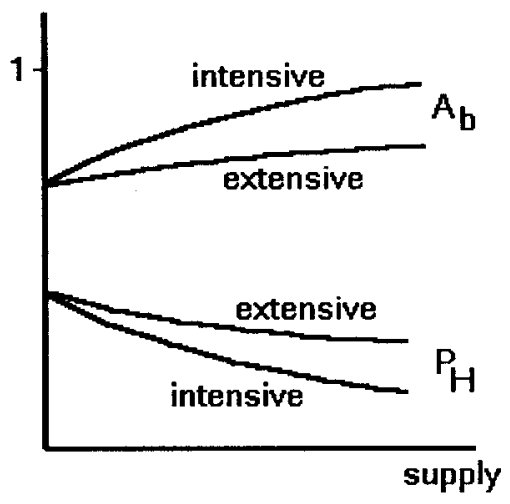

FIG. 7

$$
1-p_{L}=\frac{[(b-b x) /(1-h)]\{1-[(1-x) / m(1-h)]\}^{b m-1}}{1-\{1-[(1-x) / m(1-h)]\}^{b m}}
$$

and

$$
1-p_{H}=\frac{(b x / h)\{1-[(1-x) / m(1-h)]\}^{b m-1}}{2\left[1-(x / m h)^{b m}\right]-(b x / h)[1-(x / m h)]^{b m-1}} .
$$

Taking the limits yields (27) and (28). As indicated above, this method neglects the strategic effect that a seller can have on $U$, but in the Appendix we consider the model taking into account all relevant strategic considerations and show that the solutions generated by the two methods do indeed converge to the same limit as $m$ expands holding fixed $b=n / m$ and $h=m_{H} / m$. This is accomplished, even though we cannot actually solve for $p$ when $n$ and $m$ are finite, by showing that the equilibrium conditions for the finite model converge as $m \rightarrow \infty$ to the conditions in the model in which $U$ is taken parametrically. Q.E.D.

Figure $7 a$ depicts the effect of a change $h$ on prices, $x=m_{H} \theta_{H}$, and $A_{b}{ }^{8}$ Observe that $p_{H}$ is always above $p_{L}$, and both prices fall as $h$ goes

${ }^{8} \mathrm{~A}$ value of $b=1.5$ is used in the figure. To derive $A_{b}$ in this version of the model, note that the probability that a buyer gets served is given by $A_{b}=m_{H} \theta_{H} \Omega_{H}+m_{L} \theta_{L} \Omega_{L}$. Inserting $\Omega_{H}$ and $\Omega_{L}$ and rearranging yields

$$
A_{b}=\frac{2 h}{b}\left[1-\left(1-\frac{x}{m h}\right)^{b m}\right]-x\left(1-\frac{x}{m h}\right)^{b m-1}+\frac{1-h}{b}\left\{1-\left[1-\frac{1-x}{m(1-h)}\right]^{b m}\right\} .
$$

Taking the limit, we have

$$
A_{b} \rightarrow \frac{2 h}{b}\left[1-\exp \left(-\frac{b x}{h}\right)\right]-x \exp \left(\frac{x}{m h}\right)+\frac{1-h}{b}\left[1-\exp \left(\frac{b x-b}{1-h}\right)\right] .
$$


up, naturally. An increase in $h$ constitutes an increase in available supply along the intensive margin: there is the same number of sellers but more of them have high capacity. By contrast, a decrease in $b$ constitutes an increase in supply along the extensive margin: there are more sellers per buyer when average capacity is held fixed. Figure $7 b$ shows the difference in the impact of changes in supply along the two margins on $p_{H}$ ( $p_{L}$ looks similar) and $A_{b}$, with the increase in the total available supply kept the same. The key observation is that both variables are more responsive to supply changes along the intensive margin; that is, the price decreases more and the arrival rate for buyers increases more when we increase the number of goods per seller than when we increase the number of sellers.

Consider the implications for the typical application to the labor market, such as Mortensen and Pissarides (1994). Those models assume that the number of successful meetings between employers and workers is a function of the number of unemployed workers and the number of employers with vacancies. Thinking of employers as the analogue of sellers in our model (they post wages in exchange for jobs), we have found that it makes a difference whether there are many employers with one vacancy each or few employers with more than one vacancy. In other words, the standard specification of the matching function is incomplete if firms may post more than one vacancy. In particular, the isomatching curves (recall fig. 4) shift out as we increase the number of sellers with low capacity, holding the total number of units for sale fixed. Intuitively, frictions are more problematic when there are more locations with limited capacity.

These results imply that the number of matches will fall, given the vacancy and unemployment rates, if the firm-size distribution shifts toward more small firms. That is, the Beveridge curve (the locus of observed vacancy-unemployment pairs) will shift out as the relative number of small firms increases. It is a matter of fact that the Beveridge curve has shifted out in the postwar period, as documented by Blanchard and Diamond (1989) for the United States and by Jackman et al. (1989) for the United Kingdom. Blanchard and Diamond say that about "half [of the shift] is due to an unexplained deterministic trend" (pp. 4-5) and that the evidence suggests that "trend changes in matching, which we find in our estimation of the matching function for the period 1968 to 1981, account for a good part of this deterministic trend" (pp. 47, 50). Jackman et al. say that "shifts in the curve reflect the efficiency with which the labor market matches unemployed workers to job vacancies, and the outward shift in the UK seems mainly attributable to the fall in the effectiveness of the unemployed as job seekers" (p. 377).

Our model helps to make sense of these trend shifts to the extent that the size distribution has shifted toward smaller firms during the 
period. There is evidence to this effect at least for the 1980s (see Stanworth and Gray 1991). According to our theory, the relative increase in the number of small firms means that the efficiency of the matching technology, as a function of the vacancy and unemployment rates, is diminished. Hence, our model predicts the observed outward shift in the Beveridge curve. Of course, one would like to have more evidence for the shift in the firm-size distribution, and it would be interesting to measure quantitatively just how much this can account for the shift in the Beveridge curve, but this goes beyond the scope of the current project. $^{9}$

\section{Conclusion}

This paper has studied prices and allocations in markets with frictions. We gave a complete characterization of equilibria for the $2 \times 2$ case, including equilibria supported by triggers. However, we argued that a particular type of equilibrium-the one in which all sellers set the same price (conditional on capacity, etc.) and buyers randomize-is the interesting one on which to focus for several reasons: it is symmetric, it is robust, and it does not require an unreasonable amount of coordination. We solved for prices in this type of equilibrium for the $m \times n$ case and compared the result to the prediction of a simple alternative model. These results differ for finite $m$ and $n$ but converge to the same limit as the market grows. We derived the endogenous matching function and showed that it exhibits decreasing returns for finite $m$ and $n$, although it converges to a function with constant returns as the market grows.

We also analyzed the situation in which sellers have heterogeneous capacities. We found that prices and arrival rates for buyers are more sensitive to changes in supply generated by increasing capacity per seller than to changes in supply generated by increasing the ratio of sellers to buyers holding average capacity fixed. This means that the matching is less efficient when there are more low-capacity sellers, with total supply held constant. Intuitively, this result arises because there is a greater coordination problem among buyers when there are more small sellers (as an extreme case, if there is only one large seller, there are no frictions in our sense). Given a shift toward relatively more small employers over time, as the data suggest, we argued that the reasoning above might help to explain observed shifts in the Beveridge curve documented by various people. In future research it may be interesting to pursue this

\footnotetext{
${ }^{9}$ We do not provide an explanation here for why the size distribution has changed, or why it does not become degenerate over time, since this would requires a dynamic story beyond the scope of this paper. See Shi (2000) for a version of one such model specifically applied to the labor market. See also Julien, Kennes, and King (2000).
} 
idea quantitatively using a labor market version of the model that allows workers and firms to stay in the market for more than one period.

How much unemployment or inefficiency could be explained by such a model? Perhaps not much if a period is very short and applying for jobs is very easy: when too many workers show up at some firm today, the ones who are rationed can just go to another one tomorrow. It is plausible, however, that in some circumstances applying for jobs is not easy or costless. Consider a situation in which one has to build up capital (think of trying to get a job in an industry rather than at an individual firm) or one has to physically migrate to try to get a job in a particular region. In Steinbeck's Grapes of Wrath, people spent a lot of time and other resources moving around in search of jobs, not knowing how many other workers would be at any given location. Of course, there were other problems in that situation, and we are not suggesting that it was all a coordination failure, but simply that such coordination failures can be important and that labor market versions of models such as the one analyzed here may be interesting.

\section{Appendix}

Suppose that there are finite numbers of agents: $n$ buyers, $m_{H}$ sellers with two units, and $m_{L}=m-m_{H}$ sellers with one unit. However, here each seller takes as given the reaction functions of other agents rather than market utility $U$, as in (23) and (25). The goal is to show that the two models give the same answer when $m$ and $n$ are large.

We look for an equilibrium in which all high-capacity sellers charge $p_{H}$, all low-capacity sellers charge $p_{L}$, and all buyers go to each high-capacity seller with probability $\theta_{H}>0$ and each low-capacity seller with probability $\theta_{L}>0$, where $m_{H} \theta_{H}+m_{L} \theta_{L}=1$. The probabilities that a buyer will get served, conditional on arriving at a low- and a high-capacity seller, are given by

$$
\begin{aligned}
\Omega_{L} & =\frac{1-\left(1-\theta_{L}\right)^{n}}{n \theta_{L}}, \\
\Omega_{H} & =\frac{2}{n \theta_{H}}\left[1-\left(1-\theta_{H}\right)^{n}\right]-\left(1-\theta_{H}\right)^{n-1} .
\end{aligned}
$$

Since buyers visit all sellers with positive probability, we require $\left(1-p_{H}\right) \Omega_{H}=$ $\left(1-p_{L}\right) \Omega_{L}$, or

$$
\frac{1-p_{H}}{1-p_{L}}=\frac{\left(1 / n \theta_{L}\right)\left[1-\left(1-\theta_{L}\right)^{n}\right]}{\left(2 / n \theta_{H}\right)\left[1-\left(1-\theta_{H}\right)^{n}\right]-\left(1-\theta_{H}\right)^{n-1}}
$$

Now suppose that we are in such an equilibrium, and consider a deviation by one seller. First, consider a high-capacity seller who deviates to $p_{H}^{d}$. Let $\theta_{H}^{d}$ be the probability that a buyer visits the deviant, where

$$
\theta_{H}^{d}+\left(m_{H}-1\right) \theta_{H}+m_{L} \theta_{L}=1 .
$$

If he visits the deviant, a buyer gets served with probability 


$$
\mathbf{\Omega}_{H}^{d}=\frac{2}{n \theta_{H}^{d}}\left[1-\left(1-\theta_{H}^{d}\right)^{n}\right]-\left(1-\theta_{H}^{d}\right)^{n-1} .
$$

Given that we settle on an equilibrium in which buyers go to all sellers with positive probability, we require $\left(1-p_{H}^{d}\right) \boldsymbol{\Omega}_{H}^{d}=\left(1-p_{H}\right) \boldsymbol{\Omega}_{H}$, or

$$
\frac{1-p_{H}}{1-p_{H}^{d}}=\frac{\left(2 / n \theta_{H}^{d}\right)\left[1-\left(1-\theta_{H}^{d}\right)^{n}\right]-\left(1-\theta_{H}^{d}\right)^{n-1}}{\left(2 / n \theta_{H}\right)\left[1-\left(1-\theta_{H}\right)^{n}\right]-\left(1-\theta_{H}\right)^{n-1}},
$$

in addition to (A2).

Conditions (A3), (A5), and (A2) implicitly define a function $\theta_{H}^{d}=$ $\theta_{H}^{d}\left(p_{H}^{d} ; p_{H}, p_{L}\right)$. Taking this function and $\left(p_{H}, p_{L}\right)$ as given, the deviant highcapacity seller seeks to maximize

$$
\pi_{H}^{d}=p_{H}^{d}\left\{2\left[1-\left(1-\theta_{H}^{d}\right)^{n}\right]-n \theta_{H}^{d}\left(1-\theta_{H}^{d}\right)^{n-1}\right\} .
$$

Note that this is the same as the objective function in (25), but here the seller takes into account the reaction function $\theta_{H}^{d}=\theta_{H}^{d}\left(p_{H}^{d} ; p_{H}, p_{L}\right)$ rather than the constraint that buyers have to receive expected utility of at least $U$. An interior solution satisfies

$$
\frac{\partial \pi_{H}^{d}}{\partial p_{H}^{d}}+\frac{\partial \pi_{H}^{d}}{\partial \theta_{H}^{d}} \cdot \frac{\partial \theta_{H}^{d}}{\partial p_{H}^{d}}=0 .
$$

Similarly, consider a low-capacity seller who deviates to $p_{L}^{d}$. Let $\theta_{L}^{d}$ be the probability that a buyer visits the deviant, where

$$
\theta_{L}^{d}+m_{H} \theta_{H}+\left(m_{L}-1\right) \theta_{L}=1 .
$$

Given that we settle on an equilibrium in which buyers go to all sellers with positive probability, we require $\left(1-p_{L}^{d}\right) \Omega_{L}^{d}=\left(1-p_{L}\right) \Omega_{L}$, or

$$
\frac{1-p_{L}}{1-p_{L}^{d}}=\frac{\left(1 / n \theta_{L}^{d}\right)\left[1-\left(1-\theta_{L}^{d}\right)^{n}\right]}{\left(1 / n \theta_{L}\right)\left[1-\left(1-\theta_{L}\right)^{n}\right]},
$$

in addition to (A2). Conditions (A8), (A9), and (A2) implicitly define $\theta_{L}^{d}=$ $\theta_{L}^{d}\left(p_{L}^{d} ; p_{H}, p_{L}\right)$. Taking this and $\left(p_{H}, p_{L}\right)$ as given, the deviant low-capacity seller seeks to maximize

$$
\pi_{L}^{d}=p_{L}^{d}\left[1-\left(1-\theta_{L}^{d}\right)^{n}\right] .
$$

An interior solution satisfies

$$
\frac{\partial \pi_{L}^{d}}{\partial p_{L}^{d}}+\frac{\partial \pi_{L}^{d}}{\partial \theta_{L}^{d}} \cdot \frac{\partial \theta_{L}^{d}}{\partial p_{L}^{d}}=0 .
$$

A symmetric mixed-strategy equilibrium is a list $\left(p_{H}, p_{L}, \theta_{H}, \theta_{L}\right)$ satisfying the first-order conditions for high- and low-capacity sellers (A7) and (A11), the condition (A2), which makes buyers indifferent between visiting the two types of sellers, and the identity $m_{H} \theta_{H}+m_{L} \theta_{L}=1$, all evaluated at $\left(p_{H}^{d}, p_{L}^{d}, \theta_{H}^{d}, \theta_{L}^{d}\right)=$ $\left(p_{H}, p_{L}, \theta_{H}, \theta_{L}\right)$. This system is complicated, in general, but simplifies considerably when $m \rightarrow \infty$ with $h=m_{H} / m$ and $b=n / m$ held constant. Taking limits and inserting $\left(p_{H}^{d}, p_{L}^{d}, \theta_{H}^{d}, \theta_{L}^{d}\right)=\left(p_{H}, p_{L}, \theta_{H}, \theta_{L}\right)$, after some algebraic manipulations, we can reduce (A7) and (A11) to 


$$
\frac{p_{H}}{1-p_{H}}=\frac{-b z+2(\{[\exp (b z)-1] / b z\}-1)}{1+b z}
$$

and

$$
\frac{p_{L}}{1-p_{L}}=\frac{1-h}{b(1-h z)}\left\{\exp \left[\frac{b(1-h z)}{1-h}\right]-1\right\}
$$

where $z=\lim _{m \rightarrow \infty} m \theta_{H}$. Also, we can simplify (A2) to

$$
\frac{1-p_{H}}{1-p_{L}}=\frac{(1-h) z}{1-h z} \frac{2[1-\exp (-b z)]-b z \exp (-b z)}{1-\exp (-\{[b(1-h z)] /(1-h)\})} .
$$

These can be combined to yield one equation in $z$. Then, on solving for $z$, one can solve for $p_{H}$ and $p_{L}$. At this stage, it is a matter of routine algebra to verify that the answer is the same as the solution for the model in the text when $m \rightarrow \infty$ with $h$ and $b$ held constant.

\section{References}

Acemoglu, Daron, and Shimer, Robert. "Efficient Unemployment Insurance." J.P.E. 107 (October 1999): 893-928. (a)

. "Holdups and Efficiency with Search Frictions." Internat. Econ. Rev. 40 (November 1999): 827-49. (b)

Blanchard, Olivier J., and Diamond, Peter A. "The Beveridge Curve." Brookings Papers Econ. Activity, no. 1 (1989), pp. 1-60.

Burdett, Kenneth, and Mortensen, Dale T. "Wage Differentials, Employer Size, and Unemployment." Internat. Econ. Rev. 39 (May 1998): 257-73.

Butters, Gerard R. "Equilibrium Distributions of Sales and Advertising Prices." Rev. Econ. Studies 44 (October 1977): 465-91.

Cao, Melanie, and Shi, Shouyong. "Coordination, Matching, and Wages." Canadian J. Econ. 33 (November 2000): 1009-33.

Coles, Melvyn G., and Eeckhout, Jan. "Efficient Job Allocation." Manuscript. Philadelphia: Univ. Pennsylvania, 2000. (a)

. "Indeterminacy and Directed Search." Manuscript. Philadelphia: Univ. Pennsylvania, 2000. (b)

Jackman, Richard; Layard, Richard; and Pissarides, Christopher A. "On Vacancies." Oxford Bull. Econ. and Statis. 51 (November 1989): 377-94.

Julien, Benoit; Kennes, John; and King, Ian. "Bidding for Labor." Rev. Econ. Dynamics 3 (October 2000): 619-49.

Lagos, Ricardo. "An Alternative Approach to Search Frictions." J.P.E. 108 (October 2000): 851-73

Lang, Kevin. "Persistent Wage Dispersion and Involuntary Unemployment." Q.J.E. 106 (February 1991): 181-202.

McAfee, R. Preston. "Mechanism Design by Competing Sellers." Econometrica 61 (November 1993): 1281-1312.

Moen, Espen R. "Competitive Search Equilibrium.” J.P.E. 105 (April 1997): 385-411.

Montgomery, James D. "Equilibrium Wage Dispersion and Interindustry Wage Differentials." Q.J.E. 106 (February 1991): 163-79.

Mortensen, Dale T., and Pissarides, Christopher A. "Job Creation and Job Destruction in the Theory of Unemployment." Rev. Econ. Studies 61 (July 1994): 397-415. 
Mortensen, Dale T., and Wright, Randall. "Competitive Pricing and Efficiency in Search Equilibrium." Internat. Econ. Rev. (in press).

Peters, Michael. "Ex Ante Price Offers in Matching Games Non-steady States." Econometrica 59 (September 1991): 1425-54.

. "Limits of Exact Equilibria for Capacity Constrained Sellers with Costly Search.” J. Econ. Theory 95 (December 2000): 139-68.

Pissarides, Christopher A. Equilibrium Unemployment Theory. Oxford: Blackwell, 1990.

Rogerson, Richard. "Indivisible Labor, Lotteries and Equilibrium.” J. Monetary Econ. 21 (January 1988): 3-16.

Shell, Karl, and Wright, Randall. "Indivisibilities, Lotteries, and Sunspot Equilibria." Econ. Theory 3 (January 1993): 1-17.

Shi, Shouyong. "Product Market and the Size-Wage Differential." Manuscript. Kingston, Ont.: Queen's Univ., 2000.

Shimer, Robert. "Essays in Search Theory." Ph.D. dissertation, Massachusetts Inst. Tech., 1996.

Stanworth, John, and Gray, Colin, eds. Bolton 20 Years On: The Small Firm in the 1990s. London: Chapman, 1991. 